\title{
Exploring the acceptability of, and adherence to a carbohydrate-restricted diet as self-reported by women aged 40-55 years
}

Journal of Holistic Performance | ISSN: 2463-7238 | Published: 23 January 2018

Julia Claire McPhee ${ }^{1}$, Caryn Zinn ${ }^{1}$, Melody Smith²

jmcphee@aut.ac.nz

\begin{abstract}
Background. It is well recognised that ageing in females is associated with a predilection for weight gain. Women with abdominal obesity are known to be at especially high risk of cardiovascular and metabolic disease, as well as associated poorer overall health outcomes. Many of these metabolic disorders, however, can be alleviated through weight loss.

Methods. This research describes an exploratory study investigating the self-reported acceptance of, and adherence to, a carbohydrate-restricted diet in women aged 40-55 years old. The 8-week weight loss dietary intervention was based on a whole food approach, which was lower in total carbohydrate and higher in dietary fat than mainstream nutrition guidelines. The two primary outcome measures of this research were barriers to and motivators for acceptance of and adherence to this way of eating. The mixed methods approach employed endorsed a multi-level intervention, integrating behaviour change models with both the medium of delivery and required dietary modifications.

Hypothesis. The hypothesis underpinning this study is that a lower carbohydrate, higher fat (LCHF) dietary approach, through its macronutrient profile, has the potential to enhance an individual's selfreported adherence to behavioural lifestyle modifications required to reduce weight and improve metabolic health outcomes.
\end{abstract}

Results. Support by family members, in particular, spouses, was a key theme and appeared to act as both a barrier and motivator to adherence. Subsequent themes included satiety or feelings of fullness, being prepared by ensuring suitable food options were readily available, and acceptability of new food options.

Discussion. The findings from this study suggest self-reported adherence to this way of eating was achieved via improved satiety and enhanced social and spousal support. Moreover, results showed that for this population group, the intervention design was appropriate and medium of delivery was effective. 


\section{Background}

Global overweight and obesity rates have more than doubled in the last three decades, with 1.4 billion adults over the age of 20 years classified as overweight (body mass index [BMI] 25-29.9 $\mathrm{kg} / \mathrm{m}^{2}$ ) or obese $\left(\mathrm{BMI} \geq 3 \mathrm{O} \mathrm{kg} / \mathrm{m}^{2}\right)$ by the World Health Organisation, ${ }^{1}$ in 2008. While it is wellrecognised that comparable numbers of females and males are classified as overweight ( $35 \%$ versus $34 \%$, respectively), more women than men are categorised as obese $(14 \%$ versus $10 \%$, respectively). ${ }^{2}$

Women classified as overweight or obese exhibit higher rates of metabolic irregularities including hypertension, diabetes, and reduced mental health and energy levels than their normal/underweight counterparts. ${ }^{3}$ The prevalence of central adiposity and features of metabolic syndrome in many peri-menopausal and menopausal women transpire as a result of a reduction in oestrogen. 4 Fortunately, many of the metabolic disorders attributable to decreased oestrogen levels (exhibited in conditions such as abdominal adiposity, insulin resistance, and dyslipidaemia) can be alleviated through weight loss. Furthermore, it is understood that oestrogen has a modulating effect on the hormone insulin and lipoprotein lipase (LPL; an enzyme responsible for breaking down triglycerides), both of which are recognised as impacting central adiposity. ${ }^{5}$ During this period of mid-life weight gain and increased metabolic risk for women, preventing gains in body fat and maintaining healthy weight status is essential. ${ }^{6}$

Traditional "best practice" weight loss dietary treatment (undertaken in both research and practice) is largely based on calorie (or energy) restriction. In general, weight-loss interventions (predominantly based on traditional weight-loss guidelines) are having little impact on either global obesity rates or sustained levels of weight loss at a population level. Furthermore, middle-aged women continue to fare poorly in a life stage in which weight-gain appears unavoidable. As central weight gain with menopause is associated with the development of insulin resistance, ${ }^{6}$ there is increasing interest in weight-loss treatment options based on a macronutrient profile that elicits a reduced insulin response. In contrast to traditional "best practice," an alternative weight loss strategy promoting a model of eating that is lower in carbohydrate than mainstream guidelines (i.e. $<45 \%$ of total energy, the minimum threshold for carbohydrate), moderate in protein, and higher in fat has been posited as an effective weightloss option. This strategy recognises that the macronutrient composition of the diet itself may have a positive impact on weight loss due to the interaction between the nutrients themselves and hormonal expression.

While there remains no consensus over the most effective weight-loss strategy, it is recognised that both the traditional low fat and the more contemporary low carbohydrate, high fat (LCHF) dietary strategies are successful in eliciting weight loss at an individual level.7 However, there is evidence to suggest that LCHF diets can be successful when compared with "best practice" when it comes to improving health outcomes for individuals. Findings across several randomised controlled trials (RCTs) addressing both weight loss and health outcomes show that LCHF diets are at least as effective as low-fat diets in achieving weight loss, and more effective for improving several blood markers, in particular triglycerides and HDL cholesterol, and HbA1C (in diabetics). ${ }^{8-11}$ 
In one such study participants were randomly assigned to one of four dietary regimes. 9 In this study, women assigned to the lowest carbohydrate intake lost more weight and experienced more favourable overall metabolic effects at 12 months. Secondary analyses examined the relationship between insulin resistance and dietary adherence to either a low-fat or low-carbohydrate diet.12 Findings showed that participants were less likely to adhere to, and lose weight on, a low-fat diet if they were insulin resistant compared to those that were insulin sensitive. The authors stated that while the mechanisms explaining the reasons for this are largely unexplored, participants with insulin resistance may feel less satiated on a low-fat diet and therefore may experience stronger metabolically driven urges to consume more food. These results are compelling as they suggest that insulin resistance status may affect dietary adherence to weight loss diets. This may result in higher rates of recidivism and diminished weight loss success in insulin-resistant participants following low-fat diets. ${ }^{12}$

While weight loss in the short term appears to occur regardless of the type of diet applied, long-term sustainability of weight loss creates a greater challenge. A further strategy that affects adherence to weight loss interventions is the application of Health Behaviour Theory (HBT) in intervention design.13 A recent study designed to predict weight loss applied four leading health behaviour change theories: Social Cognitive Theory (SCT), TransTheoretical Model (TTM), Theory of Planned Behaviour (TPB) and Self Determination Theory (SDT). ${ }^{14}$ Weight change was significantly predicted by each of the HBTs analysed. It is well-recognised by health researchers, however, that no theory will be the perfect fit for any single health-based intervention and it has become evident that the use of a mixed methods approach to applying HBT to interventions elicits more favourable outcomes than interventions aimed at one level of behaviour. ${ }^{15}$

Effective weight-loss strategies, targeting priority populations through appropriate media, remain a critical public health priority and warrant ongoing investigation. ${ }^{16}$ While attrition rates in weight-loss programmes are known to be high, increased adherence to a programme can lead to successful and sustained weight loss.17 The aim of this programme is to determine factors affecting women aged 40-55 years in modifying and maintaining dietary behaviours while undertaking an LCHF way of eating for weightloss purposes.

\section{Methods}

This was a cross-sectional study investigating the self-reported acceptance of, and adherence to, a diet restricted in carbohydrate in women aged 40-55 years. The intervention itself was called the LCHF Women's Health Research Programme. The primary outcome measures of this study were barriers to and motivators for acceptance of and adherence to this way of eating. Secondary outcomes included mood state, adherence, satiety levels, and weight loss. During the intervention, weekly questionnaires were distributed to participants, starting at baseline, with the final questionnaire administered at eight weeks (end of intervention). Focus groups were conducted following completion of the intervention. Ethical approval to conduct the study was 
provided by the host institution (AUTEC reference 13/339, $14^{\text {th }}$ January 2014).

\section{Intervention}

The internet-based intervention comprised an eight-week programme designed to determine factors affecting women aged 40-55 years in modifying dietary behaviours and maintaining those behaviours while undertaking a carbohydrate-restricted way of eating. Participants were not provided with a prescription of calories or macronutrient breakdown, but rather a range of foods that aligned with the LCHF dietary approach. However, it was anticipated that food choices endorsed reflected an overall daily macronutrient profile comprising moderate amounts of protein (approximating mainstream nutrition guidelines of between 15$25 \%$ of total energy intake), reduced carbohydrate (approximating $<45 \%$ of total energy from carbohydrate), and moderate-tohigh amounts of fat (remaining calories). This macronutrient profile is considered to be a restricted carbohydrate diet, anticipated to induce weight loss in participants. ${ }^{18}$ Food options that were limited included cereals and grains, starchy vegetables and legumes and those endorsed included non-starchy vegetables, some fruit, all meats, eggs, full-fat dairy product, nuts, seeds, and cooking fats (olive oil, coconut oil, butter).

Participants were provided with access to the LCHF Women's Health Research website, on completion of the baseline questionnaire. Resources appearing on the website included detailed guidelines on food choices within the spectrum of the LCHF wholefood nutrient profile, food lists, and recipes. The website was created as a medium for delivery of information and resources and to act as a platform for communication between the lead author and participants. Resources were designed and delivered to be consistent with findings from an evidence base of research into web-based weight-loss programmes. Furthermore, the web-based nature of the programme delivery allowed for flexibility in time and venue for access to information

Further information relevant to the target population included: (i) 'tips and tricks', (ii) LCHF recipes, (iii) basic eating plans, and (iv) lists outlining foods to include and avoid. Consideration was made to weekly participant data with subsequent posts adapted to reflect themes emerging each week. The resources were designed to provide support at a level that addressed the diversity of participant needs and knowledge and aligned with the current 'stage of change'.

\section{Participants}

Participants were eligible to participate if they met the following criteria: (i) were female, (ii) were aged between 40 and 55 years, (iii) had a BMI status of overweight or obese (25 > 36 $\left.\mathrm{kg} / \mathrm{m}^{2}\right)^{1}$, (iv) were not currently following any prescribed weight-loss programme, (v) were weight-stable (weight had remained steady for the past 6 months), and (vi) were not already consuming a low carbohydrate diet.

An electronic poster advertising the study was sent via email through Auckland University of Technology (Auckland, NZ) university networks between February 2014 and March 2014. Screening for eligible participants was accomplished through self-reported measures of weight, height, and a 3-day food diary to assess dietary eligibility. 


\section{Questionnaire and scales used}

Questionnaires were administered using Question Pro online format. Weekly questionnaires included a Profile of Mood States Questionnaire Short Form (POMS SF), ${ }^{19}$ a Hunger/Fullness Scale, and a self-rated percentage sliding-scale score (ranging between 1 and 100) of adherence to the LCHF dietary regime supported with a food frequency questionnaire. Participants were also asked to submit self-reported body weight on a weekly basis. Participants received weekly questionnaires each Sunday afternoon through the eight-week study period and asked to complete these at a similar time each week. Participants were advised to weigh themselves at the same time each week; ideally in the morning, and prior to dressing.

\section{Profile of Mood States}

The POMS SF is a comprehensive assessment of transient and fluctuating moods 19,20 and was chosen to quantify any stress-response to the diet. The POMS SF questionnaire provides a measure of, (i) the tension/anxiety, (ii) depression, (iii) anger, (iv) vigour, (v) fatigue, and (vi) confusion, levels of the participant. Additional mood items included in the POMS SF questionnaire contribute to each of the key constructs. Individual mood items in the POMS $\mathrm{SF}$ were rated on a 5-point scale as follows: $0=$ "not at all," 1 = "a little," 2 = "moderately," $3=$ "quite a bit," and 4 = "extremely." This measure allows for assessment of fluctuating emotions and can aid in the effective evaluation of patterns of mood states within an individual. Individual moods scores for the five key constructs were calculated weekly for each participant and for the total mood disturbance score (TMDS). Negative mood states comprise constructs: (i) tension, (ii) depression, (iii) anger, (iv) fatigue, and (v) confusion. TMDS was calculated by adding the five negative mood states together and subtracting the positive mood state, vigour. ${ }^{20}$ Total negative moods scores (TNMS) were calculated by adding the sum of average negative moods states. TMDS, TNMS, and vigour data were presented as an average weekly score. Data were presented as an average across participants from baseline to programme completion.

\section{Adherence scale}

Perceived adherence to the LCHF diet by participants was monitored through a percentage scale with the aid of a food frequency questionnaire. The scale was presented in the questionnaire as a sliding scale from 0 to $100 \%$, with $0 \%$ representing no adherence and $100 \%$ representative of maximum adherence. An added suite of questions operated as a prompt for participants as to their adherence to the diet, asking them to note the number of occasions over the last week they had consumed a range of high carbohydrate foods that are not recommended on the LCHF diet. These foods included bread, pasta, rice, cereals, cakes, and sweets. Food frequency questions were based on a modified version of the 'Dietary Habits' section of the '2008/2009 NZ Adult Nutrition Survey'.21 Response options were adapted to correspond to the weekly (seven-day) questionnaire distribution.

\section{Self-reported weight}

Participants provided weekly self-reported weight status. While weight change over the study period was not statistically assessed, weight loss is a factor contributing to both adherence to weight-loss strategies, and 
maintenance of weight loss. ${ }^{22}$ For the purpose of this study, weight was not used as a primary variable but as a tool to indicate adherence and generate discussion in focus groups.

\section{Focus groups}

Groups were classified by participant selfreported adherence (low, medium, high adherence, using average self-reported adherence) to the LCHF food guidelines over the eight-week study period. While focus groups were based on the three adherence levels, if a participant was unable to attend the appropriate group for their adherence level, they were scheduled for the next convenient group. All weight-loss data are presented as a percentage weight loss to ensure standardisation in the variable across all participants.

\section{Data Analysis}

All quantitative data were analysed and presented using descriptive statistics, allowing indicative patterns to emerge. ${ }^{23}$ Data is presented in most cases as group findings. Probability statistics were not applied, as the study design did not allow for this type of analysis. Patterns emerging from weekly POMS $\mathrm{SF}$, adherence, satiety, and weight status were monitored and considered in the development of focus group schedule and classification. POMS data were presented as an average across participants from baseline to programme completion. Table 1 presents the schedule of questions generated for use in focus group discussions.
Table 1. Focus group schedule of questions

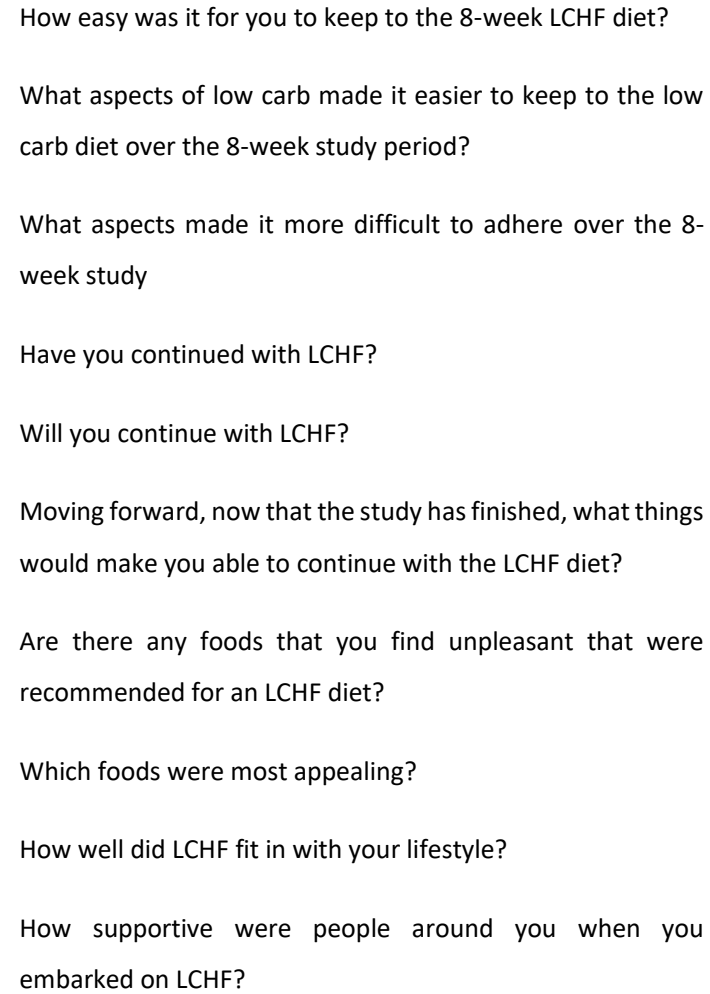

What were the challenges and confusions around what to eat on an LCHF diet?

What difference did eating low carb foods make to your budget?

Do you have any comments around how full or hungry you felt while on the LCHF diet?

Is there anything regarding the programme and resources that you would like to talk about?

Focus groups were audiotaped and transcribed. Additional hand-written notes were taken by a research officer and used as a reference document to aid with focus group transcription. Data were analysed within QSR NVivo (version 10.1.0), allowing for classification and organisation of data. ${ }^{24}$ The thematic analysis approach was used to analyse focus group data and examined themes emerging through 
participant responses. Focus group data were examined at the three categorised adherence levels: low adherence, medium adherence, and high adherence. Findings addressed participants' perceptions of the concepts impacting their ability to adhere to the LCHF diet. This process involved the evaluation of textual data and identification and coding of identified and emerging themes.

Table 2. Participant age and body size characteristics at baseline

\begin{tabular}{|c|c|c|c|}
\hline Participant & Age & Weight & BMI \\
\hline 1 & 40 & 80.2 & 29.4 \\
\hline 2 & 46 & 94.5 & 37.3 \\
\hline 3 & 53 & 67.2 & 25.1 \\
\hline 4 & 53 & 79.5 & 31.2 \\
\hline 5 & 51 & 79.1 & 25.8 \\
\hline 6 & 51 & 91.4 & 31.5 \\
\hline 7 & 52 & 77.9 & 31.4 \\
\hline 8 & 49 & 87 & 36.9 \\
\hline 9 & 53 & 108.2 & 34.9 \\
\hline 10 & 52 & 75.3 & 25.1 \\
\hline 11 & 54 & 64.4 & 25.1 \\
\hline 12 & 46 & 86.1 & 33.6 \\
\hline 13 & 54 & 104 & 34.7 \\
\hline 14 & 45 & 78.7 & 29.2 \\
\hline 15 & 51 & 80.8 & 28.7 \\
\hline
\end{tabular}

Note: $\mathrm{BMI}=$ body mass index, expressed in $\mathrm{kg} / \mathrm{m}^{2}$

\section{Results}

In total, eighteen women expressed interest in participating in the study; all were issued with eligibility questionnaires, with 15 women considered eligible to participate in the study. Two respondents failed to meet the study eligibility criteria. One respondent withdrew from the study immediately post week one. Fifteen women completed the eight-week LCHF Women's Health Research programme. Three focus groups were conducted involving all 15 participants.

\section{Survey data}

Overall, mean percentage weight loss from baseline to study completion was 5.6\% (range of $2.2 \mathrm{~kg}-8.6 \mathrm{~kg}$ ) (Figure 1). The high adherence group recorded the greatest weight loss, $7 \%$ (mean of $6.2 \mathrm{~kg}$ ), concurrent with high selfreport adherence (average adherence of 92.8\%). The low and medium adherence groups $(77.4 \%$ and $80.4 \%$, adherence respectively) had a lower total weight loss (5.3\% (mean $4.2 \mathrm{~kg}$ ) and $4.7 \%$ mean $3.8 \mathrm{~kg}$ ), respectively). 


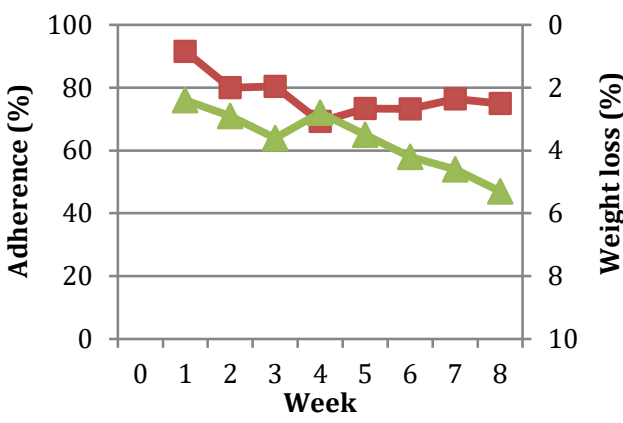

1a. Low Adherers

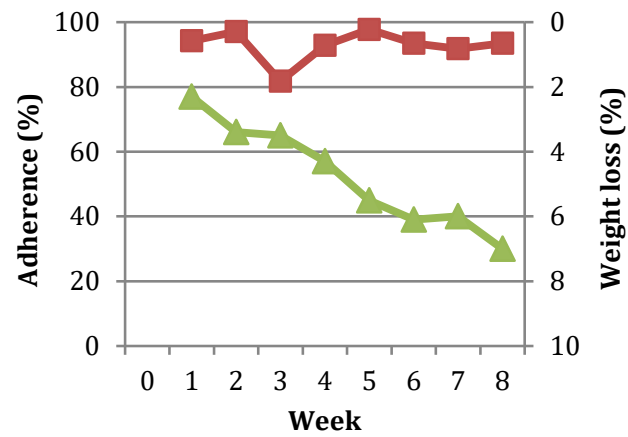

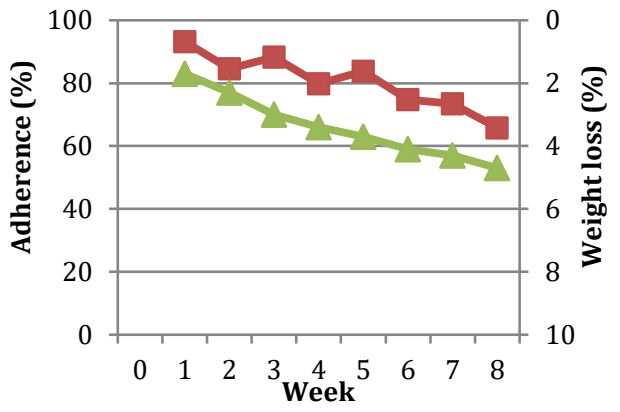

1b. Medium Adherers

1c. High adherers

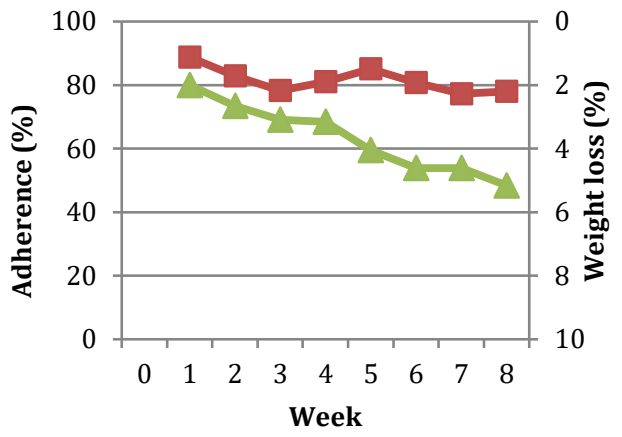

1d. Average of all participants

Figure 1: Self-reported Adherence ( $\boldsymbol{\nabla})$, and change in weight ( $\boldsymbol{\Delta})$, presented by low, medium, and high adherence level, and for all participants combined.

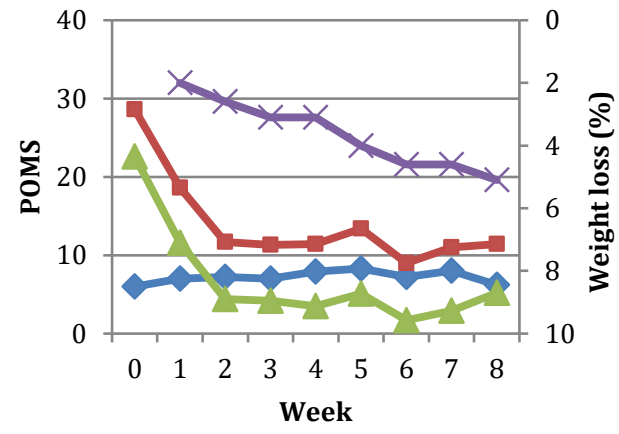

2a. POMS: Vigour, TNMS, TMDS and weight loss

Figure 2. $P O M S$ : Vigour ( $)$, TNMS ( $\boldsymbol{\bullet}), T M D S$ mood states; TMDS: Total mood disturbance, Score TNMS: Total negative mood score

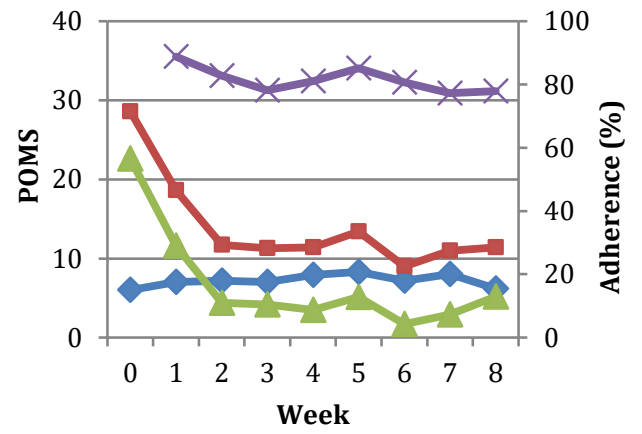

2b. POMS: Vigour, TNMS, TMDS and adherence 


\section{Focus group data}

Four over-arching themes pertaining to adherence to the LCHF model emerged from the three focus groups while undertaking the LCHF diet: (i) Importance of support from family and peers, (ii) the need to be prepared, (iii) the feeling of fullness experienced, and (iv) the attitudes to acceptability of types of food recommended on the LCHF diet.

Table 3 outlines relevant key transcripts pertaining to the each of the themes identified. Note that focus group classification was based on participant adherence level on average over the eight-week study period. The levels comprised 'Low Adherence' (mean adherence 77.4\%), Medium Adherence (mean adherence 80.4\%) and High Adherence (mean adherence $92.7 \%)$.

\section{Discussion}

Key findings from this research show that the LCHF dietary approach resulted in weight loss and improved mood states, with more weight loss noted for participants with greater adherence.

Four key themes emerged impacting adherence to these dietary modifications. Primarily, findings suggest self-reported adherence to this way of eating was achieved via a mechanism of improved satiety. In addition, both family support, and the acceptability of LCHF food options impacted participant's ability to adhere to the LCHF dietary approach.

This research presents novel findings that both align with and add to the current body of literature around factors impacting adherence and acceptability of a carbohydrate-restricted dietary approach as a weight loss option for middle-aged women. This will be achieved by determining and addressing behavioural and environmental factors that impact dietary health behaviours in the population and relating them to the existing body of research.

Descriptive data collected throughout the study highlighted some expected and unexpected findings. Percentage weight loss was greatest in participants with the highest average adherence levels across the study period. While this reflects existing findings suggesting that adherence to weight-loss interventions impacts weight-loss outcomes, ${ }^{17,25}$ as probability statistics were not applied to this data, the significance of these results remains uncertain. The overall average adherence level of $83.5 \%$, while a self-report measure, was a positive outcome and reflected focus group results around factors that impacted adherence, such as feelings of fullness, and acceptability of LCHF food options, and family support.

An interesting result of descriptive statistics displayed in the POMS data was a clear indication of the decrease in both TMDS and TNMS in the initial two weeks of the study. This study was not designed to assess causative factors of the intervention on participant mood states. However, the obvious downward trend in TMDS and TNMS is an indication that the LCHF way of eating could positively impact mood states and this characteristic of the study warrants investigation in future studies.

All themes identified could be interpreted as both a barrier and motivator to adhering to dietary modifications.

While familial support is widely acknowledged as a contributing factor to adherence to a range of positive health behaviours, adherence to dietary modifications, in particular, is dependent on family support. ${ }^{26-28}$ The current 
study findings on family support reflected those from earlier studies, whereby the support of family members emerged as a primary motivator to adhering to weight loss interventions. ${ }^{29}$, 30 Dietary factors have been shown to impact immediate family members directly, therefore willingness by family, to adapt to and include the modified food choices in their own diet can enhance participant adherence. ${ }^{28}$ Spousal involvement has been suggested as being of special importance in terms of providing emotional support for participants. ${ }^{26,31}$ Indeed, results from the current study showed that participants experienced difficulties adhering when husbands were not supportive, however participants whose husbands provided support or partook in the LCHF way of eating reported feeling motivated to adhere to the LCHF dietary approach. These results align with those reported by Hammerstrom et al. ${ }^{29}$ who found that husbands were both a major obstacle and a key motivator to adherence and behaviour change in women. In addition, when husbands refused to eat new food, requiring women to cook two meals, workload increased considerably, and created barriers to adherence to a diet. ${ }^{29}$ Similar findings were reported by Gorin, et al. ${ }^{28}$ who reported a recognition of the influence that environment holds over food choices, ${ }^{28}$ and suggested that the home environment may be an important setting to target for intervention. Given that approximately $75 \%$ of daily calories are consumed within the home environment, ${ }^{28}$ more research is warranted to determine how best to intervene and provide support in this setting. While environmental and behavioural changes that lead to weight loss and weight maintenance are required, future research should also investigate issues affecting food choices and preparation required when food is consumed outside of the home environment.

A subsequent theme, the importance of being prepared, was a theme that was also identified by Hammarström et al.29 who reported improved adherence when participants were well prepared for a range of situations. Competing demands on participants in the current study and the requirement for time spent outside of the home environment created difficulties in managing mealtimes and snacks in the workplace and social and alternate environments. The associated study website provided information to guide participants on strategies to employ when eating outside of the home, including suggestions on how to adapt mainstream food options to be LCHFappropriate. Limited choice of LCHF food options available in mainstream eateries such as cafes and bakeries, motivated some participants to create strategies to ensure LCHF food options were readily available. These actions are reflective of strategies outlined in the SDT, recognising that health behaviour is a function of characteristics of a person, their behaviour, and their environment. ${ }^{2}$ Participants who became familiar with LCHF food options and established practices so that they were prepared for all circumstances displayed motivation to adhere. It is also probable that participants developed these strategies because of HBT applied to this study through tailored resources. The study resource was developed and implemented to support women through dietary behaviour change, providing a level and depth of information that promoted participant autonomy. Drawing from components of several health behaviour theories, combining TTM eliciting stages of change principles, SCT and SDT, resources 
were tailored to an individual's current stage of change. 33 Participants were able to decide for themselves how much information they required and to what extent to access that information. Furthermore, it is apparent from these outcomes and those from previous studies that individuals with a lower selfefficacy for dietary change expect and therefore experience more barriers than motivators in daily life when intending to make a dietary change.34 For example, participants who abstained from eating or were forced to manage with available options not appropriate on the LCHF diet, increased the likelihood of periods of non-adherence. This prerequisite for preparation created a barrier, with participants reporting that dietary modifications required additional effort in already busy lives. When LCHF practices became customary, time restraints became less burdensome, and food preparation routines were easier to adhere to. This finding is in keeping with TPB, whereby a person's attitude toward a behaviour, and perceived behavioural control, combine to shape an individual's behavioural intentions and actions. 35 It must be considered that while many health behaviours require individuals to abstain permanently from a given behaviour (smoking cessation and drug abuse), food cannot be avoided completely. Therefore, dietary interventions need to be tailored to support participants to modify behaviour and sustain behaviour change in a food-dominated environment. Resources were developed in the context providing information that was easily accessible and support them to modify their behaviours in a range of situations. While individual engagement with specific resource components was not measured, participants commented that tri-weekly blog/posts added to a feeling of being supported and part of a wider group. This corresponds with research positing that participants benefit from the knowledge that they are part of a group, facing similar emotions and experiences. ${ }^{6}$

Satiety also emerged as a key theme contributing to participants' ability to adhere to the LCHF way of eating in this research. Several participants reported a satiating effect of LCHF food options, reducing the amount and frequency of food required daily. This finding corresponds to results from several studies investigating macronutrient profile of diets and the subsequent impact on satiety levels. $29,37,38$ The exact macronutrient most impacting satiety, however, remains uncertain. While some evidence suggests that the protein composition of a diet may increase satiety, 39 research by Volek et al. ${ }^{8}$ suggest that both fat and protein are equally effective in improving satiety. These findings suggest that LCHF diets could be considered a preferred weight-loss strategy to their low-fat counterpart. This study, however, was not designed to assess how individual macronutrient composition of the diet impacted satiety, but rather the impact on satiety through the combination of food choices that were higher in natural fat than participants were previously consuming. Therefore, it was not possible to know for certain whether participants were adhering to recommended levels of protein and fat, or which nutrient most impacted their satiety. High and medium adherence groups reported that eating more fat was enjoyable and contributed to the feeling of fullness. In contrast, the low adherence group indicated a reluctance to take up high-fat food options. This is a probable result of existing public health messages advising against dietary fat intake. ${ }^{21}$ It is also possible that participants in the low adherence group were often not 
satiated because of limited LCHF food options as a result of not being prepared for certain circumstances. It is well-recognised however that hunger is not the sole motivator for eating, and alternative rationale exists explaining eating habits. Eating is influenced by emotional, social and environmental factors, and individuals frequently turn to food for comfort, stress relief, or as a reward. ${ }^{40}$ Furthermore food is seen as an important and enjoyable element of social and family gatherings making habitual eating habits harder to overcome. $4^{0}$

There are several limitations to this study, one being the use of self-reported measures. Participants received weekly questionnaire each Sunday afternoon through the eight-week study period. Self-reported adherence of participants to the LCHF way of eating may have been limited by issues of recall, comprehension, and social desirability bias. To mitigate this, food frequency questions were administered to prompt recall and provide consistency. However, the reliability of responses remains uncertain. While food frequency questionnaires (FFQs) are commonly administered in intervention studies to track changes in dietary choices, it is recognised that FFQs may not be sufficiently specific to detect change. Furthermore participants may report what they consider to be the desirable responses in context of the dietary approach, ${ }^{41}$ limiting accuracy of these findings.

The introduction of food options, including those with a high-fat content previously avoided in substantial quantities, appeared to impact adherence positively. Participants' enjoyment of specific foods including Greek yoghurt, almonds, and cheese was reported with participants commenting that these food options made the LCHF diet acceptable and agreeable. It is conceivable that the fat content of these foods also contributed to satiety and the possibility of increased adherence. Furthermore, when food options endorsed in the LCHF approach were acceptable to family members, adherence was enhanced. In contrast, however, barriers were created by family members not wanting to adhere to participants' dietary modifications. Macchi et al.42 recognised that modifying the types and amounts of foods prepared in the home may be perceived by family members as a challenge to existing family norms, posing further barriers for women in adhering to weight-loss interventions.

\section{Conclusion}

The current study suggests that satiety might have an important role to play in enabling adherence to an LCHF way of eating. The findings indicate that this dietary approach was both acceptable and feasible for study participants. Moreover, results showed that for this population group, the intervention design was appropriate and medium of delivery was effective. Further investigation is required, into adherence over a longer time-frame, and into acceptability and adherence issues with other population groups. The examination of weight loss approaches for middle-aged women specifically remains a relatively novel area of research. By changing their own health behaviours to a more positive model, women can influence the dietary behaviours of family members and those around them. They are therefore a key population group to consider when designing sustainable behavioural weight-loss interventions. 
Table 3: Transcripts supporting focus group themes

\begin{tabular}{|c|c|c|c|}
\hline Theme & $\begin{array}{l}\text { Adherence } \\
\text { group }\end{array}$ & Motivators & Barriers \\
\hline \multirow{3}{*}{$\begin{array}{l}\frac{T}{0} \\
\frac{0}{2} \\
\frac{0}{0} \\
\frac{\pi}{0} \\
\frac{0}{0} \\
\infty\end{array}$} & Low & $\begin{array}{l}\text { - I was lucky because my husband did it with me, he lost more weight than me which was } \\
\text { really annoying } \\
\text { - At work we have got people who are doing it, so I have good support all around me really }\end{array}$ & $\begin{array}{l}\text { - The only culprit has been my husband. He eats chocolate and peanuts and that drawer in front of the TV comes } \\
\text { open, I tell you what it's hard when, he's sitting there munching away like that }\end{array}$ \\
\hline & Medium & $\begin{array}{l}\text { - My husband said you are much better when you don't eat carbs, you are such a psycho. No, } \\
\text { he means it in the nicest way, not quite so roller-coastering. }\end{array}$ & $\begin{array}{l}\text { - Work for me was the hard thing when people bring in chocolate and nuts } \\
\text { - She was supportive, but others were like that's a bit odd. So, then I didn't talk about it with them because they } \\
\text { were very much like 'why are you doing that? }\end{array}$ \\
\hline & High & $\begin{array}{l}\text { - I found it quite easy, easier than any diet I have tried before. Especially when the rest of the } \\
\text { family liked my meals more than their own meals. It just made it easier for me to just cook } \\
\text { the same food for everybody }\end{array}$ & $\begin{array}{l}\text { - At work they were divided because I work at a health organisation and some of them were doing it and they } \\
\text { were really keen and a couple came on board }\end{array}$ \\
\hline \multirow{3}{*}{$\begin{array}{l}\stackrel{5}{\frac{0}{\pi}} \\
\frac{0}{\pi} \\
\frac{0}{0} \\
\frac{0}{2}\end{array}$} & Low & $\begin{array}{l}\text { - I did my planning all the time, like putting my boiled eggs in the fridge and that sort of thing } \\
\text { - I brought an omelette maker to use in the microwave, at work but it's preparing it all the } \\
\text { night before } \\
\text { - I just found it was the time and preparation }\end{array}$ & $\begin{array}{l}\text { - My husband was hospitalised, and I was at the hospital for nearly a week, and I was buying the quiches at } \\
\text { muffin break and eating out and leaving the pastry because what else can you do? } \\
\text { - I just found it was the time and preparation, by the time we had had dinner, then I would have to get my lunch } \\
\text { ready and prepare my omelette to cook at work, I constantly seemed to be in the kitchen all time. }\end{array}$ \\
\hline & Medium & $\begin{array}{l}\text { - For me its planning its having a weekly menu and say right, rather than in the morning } \\
\text { scrambling for something, it's about sitting down making that plan making sure that I've got } \\
\text { low carb bread in the freezer }\end{array}$ & $\begin{array}{l}\text { - When life became busy I couldn't plan enough to be able to take snacks and food to work. That was ok but } \\
\text { that's why I would end up having toast at } 5 \text { in the morning just to keep me going and through }\end{array}$ \\
\hline & High & $\begin{array}{l}\text { - I've got to make sure I've always got nuts or something healthy in my bag. I am not going to } \\
\text { die of starvation, I can skip a meal, and yeah just making sure you are prepared. }\end{array}$ & $\begin{array}{l}\text { - You had to really think about what you were sending, and you know bread is good in the car, salads don't look } \\
\text { very nice in the car, in and out of the chilly bin }\end{array}$ \\
\hline 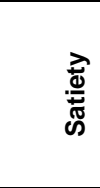 & Low & $\begin{array}{l}\text { - I was similar in that I felt fuller and I tended to eat less, I certainly didn't need to snack at all. } \\
\text { I just had breakfast lunch and dinner, and the occasional half a dozen nuts in between if I } \\
\text { needed it but not really. } \\
\text { It took a while, it took a while to get to that stage I would say, probably for me it was only in } \\
\text { the last } 3 \text { or } 4 \text { weeks where I felt I wasn't hungry, but it did take a while. }\end{array}$ & $\begin{array}{l}\text { - I still get hungry; probably I should be adding more protein into my salad at lunchtime. And maybe a bit more } \\
\text { yoghurt or something }\end{array}$ \\
\hline
\end{tabular}




\begin{tabular}{|c|c|c|c|}
\hline & Medium & $\begin{array}{l}\text { - The fact that I never got hungry. I was amazed at how I didn't get hungry when I was really } \\
\text { sticking to it. And I just didn't want to eat snacks and things, and when you were putting } \\
\text { things } \\
\text { Just not getting hungry was really interesting to me. I thought I've had hardly much to eat, } \\
2 \text { eggs for breakfast and I wouldn't even be thinking of food and that was } 7.00 \text { am to } 1.00 p m \\
\text { and I was like maybe I should be eating. }\end{array}$ & N/A \\
\hline & High & $\begin{array}{l}\text { - And now I come home at } 3 \text { and I have an egg, or I have a handful of nuts, or actually I am } \\
\text { not very hungry. I am not very hungry. } \\
\text { Definitively not hungry and before it was like full, starving, full starving, full starving and } \\
\text { frequently, whereas now it's like ok I'll have a little bit of something. So, you don't get that } \\
\text { oh I'm starving anymore. }\end{array}$ & N/A \\
\hline \multirow{3}{*}{ 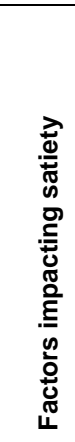 } & Low & 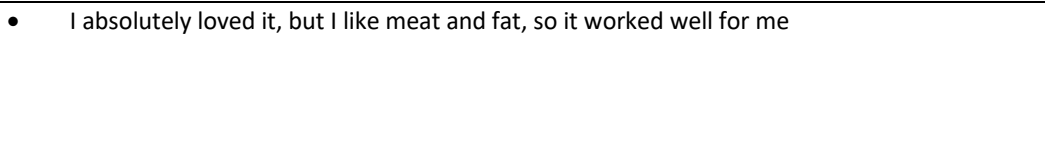 & $\begin{array}{l}\text { - It's just a bit of a shift for me because everyone has been going low fat low fat low fat and then all of a sudden } \\
\text { going to this shift and thinking it's a funny thing } \\
\text { I struggled with trying to put the high fat in, some of the fat I love, I love the fat on the pork chop, delicious, } \\
\text { chicken skin, difficult I can knock that on the head, so the low carb stuff was fine }\end{array}$ \\
\hline & Medium & $\begin{array}{l}\text { - Eating fat was great. I don't need carbs! In addition, we would go out and it would be 'can I } \\
\text { have a trim latte' and I was like 'can I have that with cream?' }\end{array}$ & $\mathrm{N} / \mathrm{A}$ \\
\hline & High & $\begin{array}{l}\text { - I found it very easy because you could have the high fat you didn't feel like you missed out } \\
\text { on anything }\end{array}$ & $\begin{array}{l}\text { - Hardest thing for people is they don't believe you can eat the high fat and lose the weight } \\
\text { - Yes, you get a bit shocked when you realise that you had 70gm of fat in that day }\end{array}$ \\
\hline \multirow{3}{*}{ 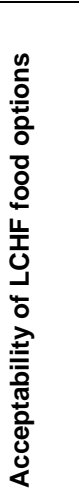 } & Low & $\begin{array}{l}\text { - I love having an omelette for breakfast, the Greek yoghurt, and the nuts that's quite nice } \\
\text { too. } \\
\text { - The nut cereal, I have that for dessert. I have Greek yoghurt cream a handful of berries that } \\
\text { I have warmed up. It's really nice. }\end{array}$ & $\mathrm{N} / \mathrm{A}$ \\
\hline & Medium & $\begin{array}{l}\text { - The Greek yoghurt with LSA and berries is just like ice cream. } \\
\text { - But now I just cut the cheese slices and make that my sandwich. Cheese is the outside of my } \\
\text { sandwiches }\end{array}$ & $\mathrm{N} / \mathrm{A}$ \\
\hline & High & $\begin{array}{l}\text { - I just to know I can get some meat with some veggies or a salad with some nice dressing on } \\
\text { it and the granola and cheese and it was all good } \\
\text { - We are eating whole naturally processed foods like cheese, like cream like meat, you know } \\
\text { coconut, so you are eating things that are natural }\end{array}$ & N/A \\
\hline
\end{tabular}




\section{Abbreviations}

Low Carbohydrate, High Fat (LCHF); Health Behaviour Theory (HBT); Social Cognitive Theory (SCT); TransTheoretical Model (TTM); Theory of Planned Behaviour (TPB); Self Determination Theory (SDT); Profile of Mood States Questionnaire Short Form (POMS SF); Total Mood Disturbance Score (TMDS); Total Negative Moods Scores (TNMS); Food Frequency Questionnaires (FFQs)

\section{Acknowledgements}

The LCHF Women's Health Research Project forms the basis of the lead author's master's thesis in Public Health. JM was supported by the Human Potential Centre, AUT throughout the development and design of this study.

\section{Author affiliations \\ ${ }^{1}$ Human Potential Centre, Auckland University of Technology, Auckland, NZ \\ ${ }^{2}$ School of Nursing, The University of Auckland, Auckland, NZ \\ Conflict of interest}

The authors declare that they have no conflict of interest

\section{Authors' contribution}

JM conceived the LCHF Women's Health Research Programme, participated in its design and coordination, conducted analysis, and drafted the manuscript.

CZ provided support regarding nutritional context of the study

MO contributed significantly to research design and analysis of data

Received: Accepted: Published:

\section{Copyright and licenses}

This work is licensed under the Creative Commons Attribution 4.0 International License. To view a copy of this license, visit

http://creativecommons.org/licenses/by/4.0/ or send a letter to Creative Commons, PO Box 1866, Mountain View, CA 94042, USA.

\section{References}

1. World Health Organization. BMI Classification 2006 [cited 2014 3rd September]. Available from: http://apps.who.int/bmi/index.jsp?introPage=intro 3.ht $\mathrm{ml}$.

2. World Health Organization. Obesity and overweight 2013 [cited 2013. Available from: http://www.who.int/mediacentre/factsheets/fs311/en/i ndex.html.

3. Brown WJ, Bryson L, Byles JE, Dobson AJ, Lee

C, Mishra G, et al. Women's Health Australia:

Recruitment for a national longitudinal cohort study. Women Health. 1998;28(1):23-40.

4. Carr MC. The emergence of the metabolic syndrome with menopause. J Clin Endocrinol Metab2003. p. 2404-11.

5. Ferland A, Château-Degat M-L, Hernandez TL, Eckel RH. Tissue-specific responses of lipoprotein lipase to dietary macronutrient composition as a predictor of weight gain over 4 years. Obesity. 2012;20(5):1006-11. 6. Davis SR, Castelo-Branco C, Chedraui P, Lumsden MA, Nappi RE, Shah D, et al. Understanding weight gain at menopause. Climacteric: Journal of the International Menopause Society. 2012;15(5):419-29.

7. Hu T, Mills KT, Yao L, Demanelis K, Eloustaz M, Yancy WS, et al. Effects of low-carbohydrate diets versus low-fat diets on metabolic risk factors: A meta-analysis of randomized controlled clinical trials. Am J Epidemiol. 2012;176(S7):S44-S54.

8. Dansinger ML, Gleason JA, Griffith JL, Selker HP, Schaefer EJ. Comparison of the Atkins, Ornish, Weight Watchers, and Zone diets for weight loss and heart disease risk reduction: A randomized trial. JAMA : Journal of the American Medical Association. 2005;293(1):43-53.

9. Gardner CD, Kiazand A, Alhassan S, Kim S, Stafford RS, Balise RR, et al. Comparison of the Atkins, Zone, Ornish and LEARN diets for change in weight and related risk factors among overweight premenopausal women the $A$ to $Z$ weight loss study: A randomized clinical trial. JAMA: Journal of the American Medical Association. 2007;297(9):969-77.

10. Stern L, Iqbal N, Seshadri P, Chicano KL, Daily DA, McGrory J, et al. The effects of low-carbohydrate versus conventional weight loss diets in severely obese adults: One-year follow-up of a randomized trial. Ann Intern Med. 2004;140(10):778-85.

11. Forsythe CE, Phinney SD, Fernandez ML, Quann EE, Wood RJ, Bibus DM, et al. Comparison of low fat and low carbohydrate diets on circulating fatty acid composition and markers of inflammation. Lipids. 2008;43(1):65-77.

12. McClain AD, Otten JJ, Hekler EB, Gardner CD. Adherence to a low-fat vs. low-carbohydrate diet differs by insulin resistance status Diabetes, Obesity and Metabolism. 2013;15(1):87-90.

13. Ammerman AS, Lindquist $\mathrm{CH}$, Lohr KN, Hersey J. The efficacy of behavioral interventions to modify dietary fat and fruit and vegetable intake: A review of the evidence. Prev Med. 2002;35(1):25-41.

14. Palmeira A, Teixeira P, Branco $T$, Martins $S$, Minderico C, Barata J, et al. Predicting short-term weight 
loss using four leading health behavior change theories. International Journal of Behavioral Nutrition and Physical Activity. 2007;4(1):14-26.

15. Elder JP, Schmid TL, Dower P, Hedlund S. Community heart health programs: Components, rationale, and strategies for effective interventions. J Public Health Policy. 1993;14(4):463-79.

16. Levy RL, Finch EA, Crowell MD, Talley NJ, Jeffery RW. Behavioral intervention for the treatment of obesity: Strategies and effectiveness data. The American Journal of Gastroenterology. 2007;102(10):2314-21. 17. Greenberg I, Stampfer MJ, Schwarzfuchs D, Shai I. Adherence and success in long-term weight loss diets: The dietary intervention randomized controlled trial (DIRECT). J Am Coll Nutr. 2009;28(2):159-68. 18. Mullins $\mathrm{G}$, Hallam CL, Broom I. Ketosis, ketoacidosis and very low calorie diets: Putting the record straight. Nutrition Bulletin. 2011;36(3):397-402. 19. Shacham S. A shortened version of the Profile of Mood States. J Pers Assess. 1983;47(3):305-6.

20. McNair D, Lorr M, Droppleman L. Profile of mood states. San Diego, California. 1992;53:6.

21. Ministry of Health. Food and nutrition guidelines for healthy adults: A background paper. Ministry of Health PO Box 5013, Wellington New Zealand: Ministry of Health, 2003.

22. Alhassan S, Kim S, Bersamin A, King AC, Gardner $C D$. Dietary adherence and weight loss success among overweight women: Results from the $A$ to $Z$ weight loss study. Int J Obes. 2008;32(6):985-91. 23. Baumgartner TA, Strong $\mathrm{CH}$, Hensley LD. Conducting And Reading Research In Health and Human Performance. 4th ed: McGraw-Hill Humanities/Social Sciences/Languages; 2005.

24. Bazeley P, Jackson K. Qualitative data analysis with NVivo. 2nd ed: Sage Publications Limited; 2013. 3 p. 25. Klein S. Clinical trial experience with fatrestricted vs carbohydrate-restricted weight-loss diets. Obes Res. 2004;12(90002):S141-S4.

26. McLean N, Griffin S, Toney K, Hardeman W. Family involvement in weight control, weight maintenance and weight-loss interventions: A systematic review of randomised trials. International Journal of Obesity and Related Disorders. 2003;27(9):987-1005.

27. Gorin AA, Powers TA, Koestner R, Wing RR, Raynor HA. Autonomy support, self-regulation, and weight loss. Health psychology: Official journal of the Division of Health Psychology, American Psychological Association. 2014;33(4):332-9.

28. Gorin AA, Raynor HA, Fava J, Maguire K, Robichaud E, Trautvetter J, et al. Randomized controlled trial of a comprehensive home environment-focused weight-loss program for adults. Health Psychol. 2013;32(2):128-37.

29. Hammarström A, Wiklund AF, Lindahl B, Larsson C, Ahlgren C. Experiences of barriers and facilitators to weight-loss in a diet intervention - A qualitative study of women in northern Sweden. BMC Womens Health. 2014;14(1):59-69.

30. Visram S, Crosland A, Cording H. Triggers for weight gain and loss among participants in a primary care-based intervention. Br J Community Nurs. 2009;14(11):495-501.

31. Potz Kieffer B. The experience of obesity, its treatment and weight loss: A qualitative study of overweight couples: Doctoral dissertation, Plymouth University; 2007.

32. Baranowski T, Cullen KW, Nicklas T, Thompson $D$, Baranowski J. Are current health behavioral change models helpful in guiding prevention of weight gain efforts? Obes Res. 2003;11(10S):S23-S43.

33. Prochaska JO, Wright JA, Velicer WF.

Evaluating theories of health behavior change: $A$ hierarchy of criteria applied to the transtheoretical model. Applied Psychology: An International Review. 2008;57(4):561-88.

34. Baranowski T, Cullen KW, Baranowski J. Psychosocial correlates of dietary intake: Advancing dietary intervention. Annu Rev Nutr. 1999;19(1):17-40. 35. Glanz K, Rimer BK, Viswanath K. Health behavior and health education: Theory, research, and practice. San Francisco, CA: Jossey-Bass; 2008.

36. Glanz K. Compliance with dietary regimens: Its magnitude, measurement, and determinants. Prev Med. 1980;9(6):787-804.

37. Chambers L, Yeomans MR. Individual differences in satiety response to carbohydrate and fat. Predictions from the Three Factor Eating Questionnaire (TFEQ). Appetite. 2011;56(2):316-23.

38. Volek JS, Westman EC. Very-low-carbohydrate weight-loss diets revisited. Cleve Clin J Med.

2002;69(11):849, 53, 56.

39. Paddon-Jones D, Westman E, Mattes RD, Wolfe RR, Astrup A, Westerterp-Plantenga M. Protein, weight management, and satiety. The American Journal of Clinical Nutrition. 2008;87(5):1558S-61S.

40. Katterman SN, Kleinman BM, Hood MM, Nackers LM, Corsica JA. Mindfulness meditation as an intervention for binge eating, emotional eating, and weight loss: A systematic review. Eating Behaviors. 2014;15(2):197-204.

41. Cade J, Thompson R, Burley V, Warm D. Development, validation and utilisation of foodfrequency questionnaires - A review. Public Health Nutr. 2002;5(4):567-87.

42. Macchi C, Russell C, White M. Shifting processes model: A conceptual model for sustainable weight management. Families, Systems, \& Health. 2013;31(4):326-37. 